\title{
Degradation of Volatile Hydrocarbons Using Continuous-Flow Photocatalytic Systems with Enhanced Catalytic Surface Areas
}

\author{
Wan-Kuen Jo ${ }^{1 \dagger}$, Sung-Hoon Yang ${ }^{1}$, Seung-Ho Shin ${ }^{1}$, Sung-Bong Yang ${ }^{2}$ \\ ${ }^{1}$ Department of Environmental Engineering, Kyungpook National University, Daegu 702-701, Korea \\ ${ }^{2}$ Department of Chemistry, College of Natural Science, University of Ulsan, Ulsan 680-749, Korea
}

\begin{abstract}
Limited information is available on the degradation of volatile hydrocarbons determined via the use of plate-inserted photocatalytic reactors. This has led to the evaluation of surface areas of cylindrical continuous-flow photocatalytic reactors for the degradation of three selected aromatic hydrocarbons. Three types of reactors were prepared: a double cylinder-type, a single cylindrical-type without plates and a single cylindrical-type with inserted glass tubes. According to diffuse reflectance, FTIR and X-ray diffraction (XRD) spectroscopy, the surface characteristics of a coated photocatalyst were very similar to those of raw $\mathrm{TiO}_{2}$, thereby suggesting that the coated photocatalyst exhibited the same photocatalytic activity as the raw $\mathrm{TiO}_{2}$. The photocatalytic degradation efficiencies were significantly or slightly higher for the single cylinder-type reactor than for the double cylinder-type reactor which had a greater catalytic surface area. However, for all target compounds, the degradation efficiencies increased gradually when the number of plates was increased. Accordingly, it was suggested that the surface area being enhanced for the plate-inserted reactor would elevate the photocatalytic degradation efficiency effectively. In addition, this study confirmed that both initial concentrations of target compounds and flow rates were important parameters for the photocatalytic removal mechanism of these plate-inserted photocatalytic reactors.
\end{abstract}

Keywords: Double cylinder-type, Operational condition, Plate, Spectroscopy, Single cylinder-type

\section{Introduction}

Volatile hydrocarbons which are present in large quantities in multiple environments have been the recipient of a great deal of work mainly because of their toxic effects on human health. In particular, aromatic compounds such as toluene, ethyl benzene and xylenes have been detected at high concentration levels in both industrial and residential environments, mainly resulting from anthropogenic sources such as motor vehicle exhausts and other combustion processes utilizing fossil fuels, petroleum storage and distribution, solvent usage as well as other industrial processes [1,2]. These compounds are classified as hazardous air pollutants, which can cause a variety of health problems such as cancerous illnesses, respiratory irritation and central nervous system damage $[3,4]$. In addition, they are closely associated with a variety of types of DNA damage such as single-strand breaks, double-strand breaks and oxidative base modification with neurotoxicity as well as hepatotoxicity $[5,6]$. These characteristics of aromatic hydrocarbons led to the development of control measures for reducing health and environmental risks from exposure to these pollutants.
Photocatalysis has become a highly promising technology for the removal of various environmental pollutants [7-9]. In particular, this technology has the potential to degrade a broad range of volatile hydrocarbons to non-toxic compounds $\left(\mathrm{CO}_{2}\right.$ and $\mathrm{H}_{2} \mathrm{O}$ ). This can be achieved as a result of reactions either with molecular oxygen or with hydroxyl radicals and super-oxide ions. These super-oxide ions are form after the initial production of highly reactive electron and hole pairs, which occurs when the photocatalysts are ultraviolet (UV) irradiated [7, 10, 11]. The operational efficiency of this technology for environmental pollutants depends upon several photocatalytic conditions [10]. The surface area of photocatalysts is an important parameter in the photocatalytic degradation processes of environmental pollutants [10, 12]. Based on theoretical, numerical and experimental studies, Mo et al. [12] have reported that formaldehyde degradation performance could be improved via the enhanced surface area of a photocatalytic system.

Various geometric shapes of photocatalytic reactors have been applied to the removal of volatile hydrocarbons [13-15]. Amongst these geometries, a cylindrical continuous flow type is one of the most commonly used reactors for air control applica- (c) This is an Open Access article distributed under the terms of the Creative Commons Attribution Non-Commercial License (http://creativecommons. org/licenses/by-nc/3.0/) which permits unrestricted non-commercial use, distribution, and reproduction in any medium, provided the original work is properly cited.
Received March 22, 2011 Accepted May 12, 2011

${ }^{\dagger}$ Corresponding Author

E-mail: wkjo@knu.ac.kr

Tel: +82-53-950-6584 Fax: +82-53-950-6579 
tions, as it provides a well-characterized reactive catalyst surface along the length of the reactor body and allows for uniform light distribution [13]. In addition, this geometry allows for lower reaction times when compared with batch reactors. As such, the present study was designed to evaluate the enhanced surface areas of cylindrical continuous-flow photocatalytic reactors on the degradation of three selected aromatic hydrocarbons (toluene, ethyl benzene, and xylenes). These target compounds are three of the most abundant components contained in gasoline and some of the most commonly used in industrial solvents [16]. The surface area of photocatalysts was enhanced using two different types of reactors, a double cylinder-type reactor and a single cylindrical-type reactor with inserted glass plates. For the purpose of comparison, another single cylindrical-type reactor without any inserted plates was also prepared. The present study also examined the characteristics of the coated photocatalyst using diffuse reflectance, FTIR and X-ray diffraction (XRD) spectroscopy.

\section{Materials and Methods}

\subsection{Preparation of Reactors}

Three types of reactors were prepared: a double cylinder-type, a single cylindrical-type without plates and a single cylindricaltype with inserted glass tubes. Fig 1 illustrates the double cylinder-type reactor, which consists of a cylindrical glass tube with another cylindrical glass tube with a smaller diameter placed within. Another cylindrical glass tube whose size was same as the outer cylindrical tube was used for the single cylindrical re-

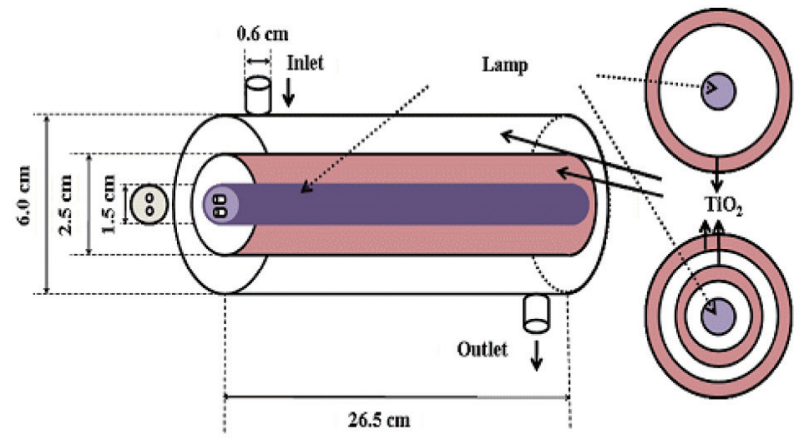

Fig. 1. Top and side views of a double cylinder-type reactor.

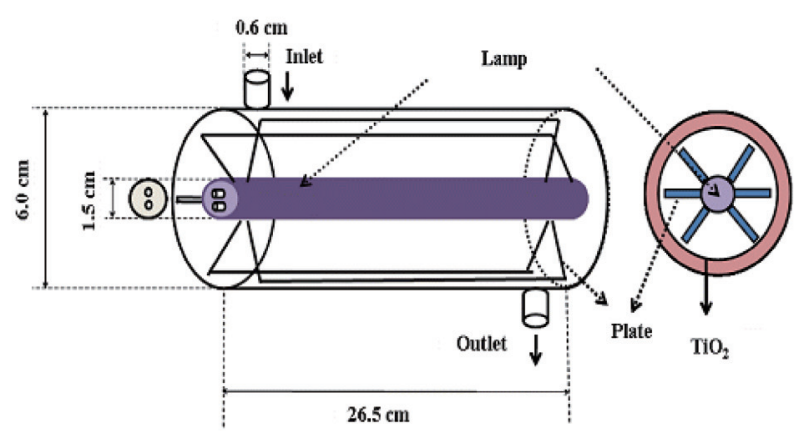

Fig. 2. Top and side views of a single cylinder-type reactor with inserted glass plates. actor without plates. Fig. 2 illustrates the single cylindrical-type with inserted glass tubes. For this type of reactor, both the inner surface of the tube and the surfaces of plates exposed to the gas flowing through the annular region were coated with thin films of $\mathrm{TiO}_{2}$ (Degussa P-25). For all reactor types, a cylindrical light source was inserted into the tube and served as the inner surface of the cylindrical reactors. The reactor was constructed to direct the incoming air flow towards the light in order to increase the air turbulence inside the reactor, thereby enhancing the distribution of the target compounds onto the catalytic surface of the reactor. The outer surface of the reactors was wrapped with aluminum foil in order to minimize the light loss through the reactor surface as well as to prevent light gain from the laboratory fluorescent lamps.

\subsection{Characterization of Photocatalyst Surface}

The surface characteristics of the coated $\mathrm{TiO}_{2}$ photocatalysts were obtained using a diffuse reflectance UV-VIS-NIR spectrophotometer, an FTIR spectrophotometer and an XRD meter. Visible absorption spectra were obtained for the dry pressed disk samples using a Varian CARY 5G spectrophotometer equipped with an integrating sphere with Polytetrafluoroethylene being used as a reference. FTIR analysis was performed on a PerkinElmer Spectrum GX spectrophotometer at a resolution of 4 $\mathrm{cm}^{-1}$ in the spectral range of 400-4,000 $\mathrm{cm}^{-1}$, using a KBR pellet for sample preparation. XRD patterns were determined on a Rigaku D/max-2500 diffractometer with $\mathrm{Cu} \mathrm{K}_{\alpha}$ radiation operated at $40 \mathrm{kV}$ and $100 \mathrm{~mA}$. The particle morphology was observed using a Hitachi S-4300 (Hitachi, Tokyo, Japan) \& EDX-350 FESEM (Hitachi) at an acceleration voltage of $15 \mathrm{kV}$.

\subsection{Survey Protocols}

Two experiments were conducted for the evaluation of the enhanced surface areas of cylindrical continuous-flow photocatalytic reactors for the degradation of toluene, ethyl benzene and xylenes. For the first experiment, two reactors, a single cylindrical-type reactor without plates and a double cylinder-type reactor, were tested for their photocatalytic activity. The major operational conditions were as follows: the initial concentration (IC) of the individual compound was $0.5 \mathrm{ppm}$; flow rate of $2 \mathrm{~L}$ $\mathrm{min}^{-1}$; relative humidity $(\mathrm{RH})$ range of $50-55 \%$; lamp type being used was a 8-W fluorescent black light lamp (SANKYO DENKI F8T5/BLB; Sankyo Denki, Tokyo, Japan) with a maximum spectral intensity at $352 \mathrm{~nm}$. Under these operational conditions, a second experiment was performed to examine the photocatalytic characteristics of another two reactors, a single cylindricaltype reactor without plates and a single cylindrical-type reactor with six inserted plates. In addition, the single cylindrical-type reactor with inserted plates was investigated for its photocatalytic activity under various operational conditions. The photocatalytic operational conditions were determined on the basis of three operational parameters, the number of plates, ICs and the flow rates. The number of plates used was either $0,3,6$, or 9 . The ICs used included $0.1,0.5,10,50,75$, and $100 \mathrm{ppm}$, in order to cover both residential and industrial levels. The range of air flow rates investigated was from 0.4 to $2.3 \mathrm{~L} \mathrm{~min}^{-1}$. All other parameters were fixed to their representative values: the number of plates, 6; IC, $0.1 \mathrm{ppm}$; flow rate, $1 \mathrm{~L} \mathrm{~min}^{-1}$; RH range, 50-55\%. However, the representative IC used was $1.0 \mathrm{ppm}$ for the experiment conducted to examine the effect of the fin number on deg- 
radation efficiency.

The major experimental parameters that required control included control of RH, ICs and flow rates. Humidified air was generated by passing zero-grade air through a charcoal filter followed by a humidification device in a water bath (Cole-Parmer HAAKE W26; Cole-Parmer Inc., Vernon Hills, IL, USA). The RH is measured just in front of the PCO reactor inlet using a humidity meter (Thermo Recorder TR-72S; T \& D Co., Tokyo, Japan). The flow rate measurements were carried out using identical rotameters $\left(0-10 \mathrm{~L} \mathrm{~min}^{-1}\right)$ calibrated against a dry test meter (URG 3000-020C). The humidified air stream then flowed into a Pyrex mixing bulb where it was mixed with the standard VOCs, which were then injected into the bulb via a syringe pump (KdScientific Model 210; KdScientific, Holliston, MA, USA). The air stream was then finally fed into the PCO reactor.

\subsection{Measurements}

Target compounds in the air stream were collected by filling an evacuated $5 \mathrm{~L}$ Tedlar bag at a constant flow rate. Air from this bag was then drawn through a sorbent trap containing 0.3 g of Tenax TA using a constant flow sampling pump (Model 224-PCXR4; SKC Inc., Eighty Four, PA, USA). All of the samples were taken at ambient room temperature $\left(19-25^{\circ} \mathrm{C}\right)$. The target compounds collected on the sorbent trap were analyzed by coupling a thermal desorption system (Thermal Desorber SPIS-TD; Donam Instrument, Seongnam, Korea) to a gas chromatograph (Agilent 4890D; Agilent Inc., Wilmington, DE, USA) with a flame ionization detector using a $0.32 \mathrm{~mm}$ I.D. fused silica column of length $60 \mathrm{~m}$ (J \&W Scientific, Folsom, CA, USA).

The quality assurance/quality control program for measurement of the target compounds included laboratory blank traps and spiked samples. At the beginning of the day, a laboratory blank trap was analyzed to check for any contamination, however no such contamination was identified. An external standard was analyzed daily to check the quantitative response, when this differed more than $\pm 20 \%$ from that predicted by the specified calibration equation, a new calibration equation was determined. The method detection limits ranged from 1.2 to 3.3 $\mu \mathrm{g} \mathrm{m}^{-3}$, depending on isomers.

\section{Results and Discussion}

\subsection{Surface Characteristics of the Coated Photocatalyst}

The coated $\mathrm{TiO}_{2}$ photocatalyst was characterized using three spectroscopy methods. The UV-visible absorbance spectrum of the photocatalyst is presented in Fig. 3. This photocatalyst revealed maximum absorption at $\lambda<300 \mathrm{~nm}$, with the absorption edge at $\lambda \approx 430 \mathrm{~nm}$, which was similar to that reported by other studies [17-19]. $\mathrm{TiO}_{2}$ exhibits a relatively high activity only under UV light, which exceeds the band-gap energy of $3.0 \mathrm{or} 3.2 \mathrm{eV}$ in the rutile or anatase crystalline phases respectively [10]. When this photocatalyst is irradiated with photons with a wavelength of less than $385 \mathrm{~nm}$, the band gap energy is exceeded and an electron is promoted from the valence band to the conduction band [10]. Accordingly, the maximum absorption obtained at $\lambda<$ $300 \mathrm{~nm}$ confirms that the coated $\mathrm{TiO}_{2}$ can be photocatalytically activated in the same way as raw $\mathrm{TiO}_{2}$.

Fig. 4 reveals the XRD patterns of coated $\mathrm{TiO}_{2}$ calcined at $500^{\circ} \mathrm{C}$ for $3 \mathrm{hr}$. The X-ray diffractograms exhibited an anatase crystal phase with a distinct peak at $2 \theta=25.2^{\circ}$ and a rutile crystal phase with a distinct peak at $2 \theta=27.4^{\circ}$, these are consistent with the results of previous studies $[17,18]$. This finding confirms that the coated $\mathrm{TiO}_{2}$ has similar morphological characteristics to raw $\mathrm{TiO}_{2}$.

As represented in Fig. 5, major peaks in the FTIR spectrum

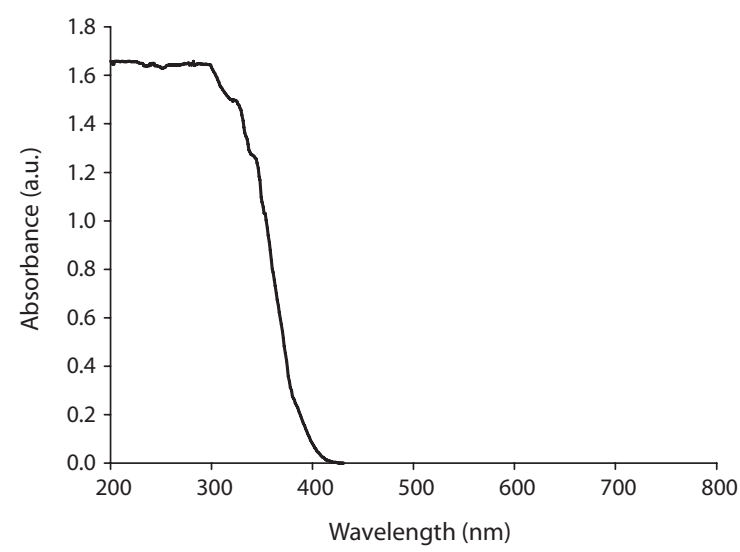

Fig. 3. Ultraviolet-visible spectra of $\mathrm{TiO}_{2}$ coated onto reactors.

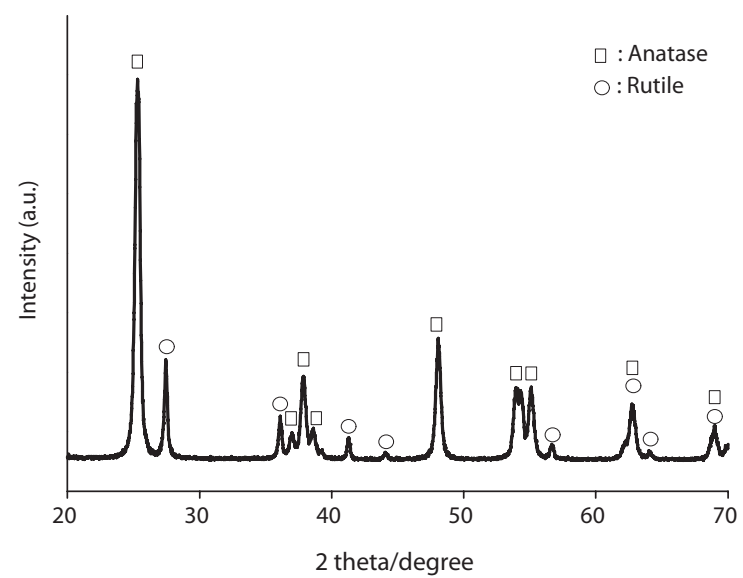

Fig. 4. X-ray diffraction (XRD) pattern of $\mathrm{TiO}_{2}$ coated onto reactors.

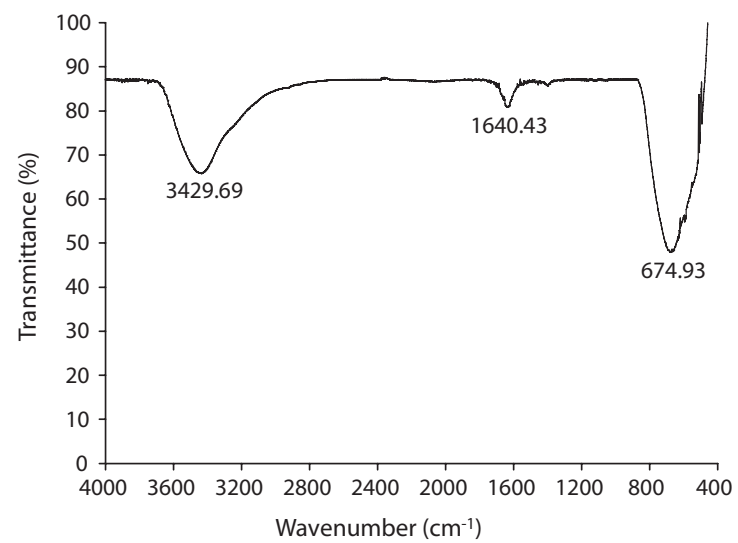

Fig. 5. Fourier transforms infrared (FTIR) spectra of $\mathrm{TiO}_{2}$ coated onto reactors. 
of the photocatalyst were located at 3,436-3,410, 1,640-1,631, and $683-675 \mathrm{~cm}^{-1}$. Similarly, Mejía et al. [20] reported four main absorption peaks located at 3,480, 1,600, 1,030 and $530 \mathrm{~cm}^{-1}$ for their $\mathrm{TiO}_{2}$ samples. The band at $3,436-3,410 \mathrm{~cm}^{-1}$ appears to be due to the $\mathrm{O}-\mathrm{H}$ stretching vibration, while the band at 1,640 $1,631 \mathrm{~cm}^{-1}$ is likely due to $\mathrm{O}-\mathrm{H}$ bending of the water molecules absorbed on the catalyst surface [21,22]. The correspondence of the band below $1,000 \mathrm{~cm}^{-1}$ could have been due to vibration of the titania crystal lattice [22]. Consequently, the surface characteristics of the coated photocatalyst were very similar to those of the raw $\mathrm{TiO}_{2}$, suggesting that the coated photocatalyst exhibited the same photocatalytic activity as raw $\mathrm{TiO}_{2}$.

\subsection{Photocatalytic Activities}

The present study evaluated the enhanced surface areas of cylindrical continuous-flow photocatalytic reactors for the photocatalytic degradation of selected aromatic hydrocarbons using three different reactor types. The destruction of the VOCs occurs as a result of reactions with the molecular oxygen or reactions with hydroxyl radicals and super-oxide ions formed after the initial production of highly reactive electron and hole pairs when the photocatalyst is light-irradiated. Fig. 6 illustrates the photocatalytic degradation efficiencies of toluene, ethyl benzene and xylenes determined via single and double cylinder-type reactors. With respect to two of the compounds, toluene and ethyl benzene, the degradation efficiencies were significantly or slightly higher for the single cylinder-type reactor $\left(499 \mathrm{~cm}^{2}\right)$ than for the double cylinder-type reactor $\left(707 \mathrm{~cm}^{2}\right)$, which had a greater catalytic surface area. The degradation efficiencies for the single cylinder-type reactor were $77 \%$ and $84 \%$ for toluene and ethyl benzene respectively, while for the double cylindertype reactor they were $53 \%$ and $78 \%$. Considering their catalytic surface areas only, the degradation efficiency for the double cylinder-type reactor ought to be higher since the photocatalytically active surface area is an important parameter [10,12]. However, the average light intensity irradiated onto the catalyst surface measured at the inner catalytic surface of the outer tube for the double cylinder-type reactor $\left(0.8 \mathrm{~mW} \mathrm{~cm}^{-2}\right)$ was much lower than that for the single cylinder-type reactor $\left(4.2 \mathrm{~mW} \mathrm{~cm}^{-2}\right)$. This is most likely due to the blocking effect of the $\mathrm{TiO}_{2}$ film coated on the outer surface of the inner tube for the double cylinder-type reactor. Light intensity is another important parameter in the photocatalytic processes of chemicals [10]. As such, the current finding suggests that the light intensity effect would outweigh the enhanced surface area effect on the photocatalytic degradation efficiencies of the target compounds. In contrast to toluene and ethyl benzene, the degradation efficiencies for xylenes were not significantly different between the single cylinder-type reactor and the double cylinder-type reactor. One possible explanation for this result is that xylenes have higher photocatalytic oxidation rates when compared to the other two compounds [23]. Since both reactors revealed high degradation efficiencies, the differences are barely distinguishable.

This work was derived to examine a new reactor having an enhanced catalytic surface area. Fig. 7 represents degradation efficiencies of toluene, ethyl benzene and xylenes determined by the number of plates via a single cylinder-type reactor with inserted glass plates whose surfaces were coated with $\mathrm{TiO}_{2}$. The experimental conditions used to obtain the data for Fig. 7 were different from those for Fig. 6, which were described in section 2.3. Survey Protocols. For all of the target compounds, the degra- dation efficiencies increased gradually when the plate number was increased. For example, the average degradation efficiencies determined via the single cylinder-type reactor with nine glass plates inserted were $73 \%, 76 \%$, and $86 \%$ for toluene, ethyl benzene and xylenes respectively, whereas those for the single cylinder-type reactor without any plates inserted were $45 \%$, $53 \%$, and $54 \%$. The elevated degradation efficiency for the former type of reactor is mainly attributed to the increased reaction area and mass transfer rate for the plate-inserted reactor [12]. Meanwhile, the light intensity measured at the inner catalytic surface of the former reactor with nine plates $\left(3.7 \mathrm{~mW} \mathrm{~cm}^{-2}\right)$ was somewhat lower than that for the latter reactor $\left(4.2 \mathrm{~mW} \mathrm{~cm}^{-2}\right)$. This is likely due to the interference in light transport caused by the plates from the lamp to the inner catalytic surface of the single cylinder-type reactor with inserted plates [12]. As such, the gradual increase in the degradation efficiency with number of plates suggests that the effects of an increased reaction area and mass transfer rate would outweigh the effects of the lightintensity decrease on the inner wall surface on the degradation efficiency.

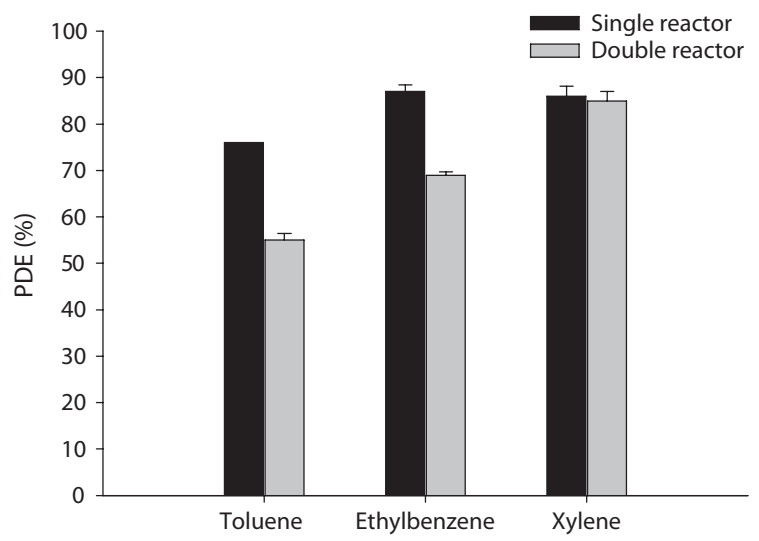

Fig. 6. Photocatalytic degradation efficiencies (PDEs, \%) of toluene, ethyl benzene and xylenes determined via single and double cylindertype reactors.

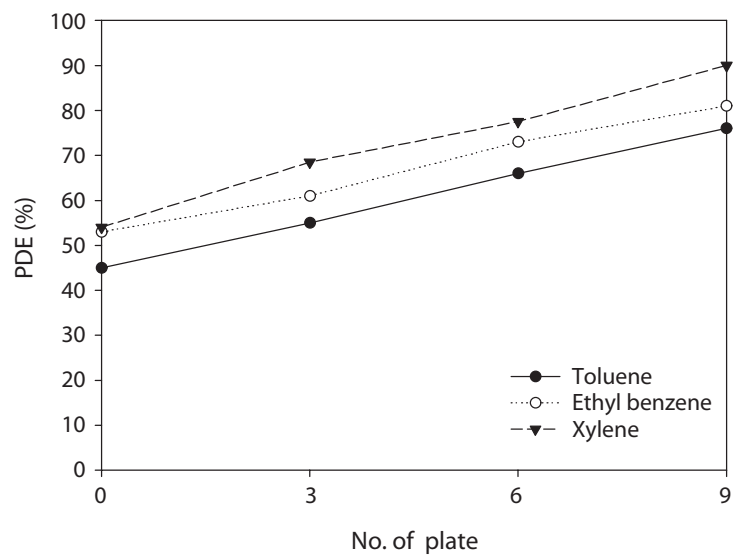

Fig. 7. Photocatalytic degradation efficiencies (PDEs, \%) of toluene, ethyl benzene and xylenes determined via a single cylinder-type reactor with inserted plates whose surfaces were coated with $\mathrm{TiO}_{2}$ against the number of plates. 
The degradation efficiencies of the target compounds according to the IC, as determined via the single cylinder-type reactor with six plates inserted are exhibited in Fig. 8. The relationships between the degradation efficiencies and ICs for toluene, ethyl benzene and xylenes respectively, are as follows:

$\mathrm{PDE}($ toluene $)=6.9 / \mathrm{IC}+22.4 \quad\left(\mathrm{R}^{2}=0.73, \mathrm{p}<0.05\right)$

PDE (ethyl benzene $)=6.6 / \mathrm{IC}+28.4\left(\mathrm{R}^{2}=0.77, \mathrm{p}<0.05\right)$

$\mathrm{PDE}($ xylenes $)=5.1 / \mathrm{IC}+45.7 \quad\left(\mathrm{R}^{2}=0.62, \mathrm{p}<0.05\right)$

Where PDE represents the photocatalytic degradation efficiencies expressed as a percentage, which exhibit a decreasing trend with the increase in IC. When the IC is increased from 0.1 to 100 ppm, the average degradation efficiencies decreased from $86 \%$ to $8 \%$, from $88 \%$ to $11 \%$ and from $92 \%$ to $27 \%$ for toluene, ethyl benzene and xylenes respectively. Similarly, previous studies [24-26] also reported this IC dependence on the removal efficiency of several volatile hydrocarbons, as determined via $\mathrm{TiO}_{2}$ systems without any plates inserted. This IC dependence is most

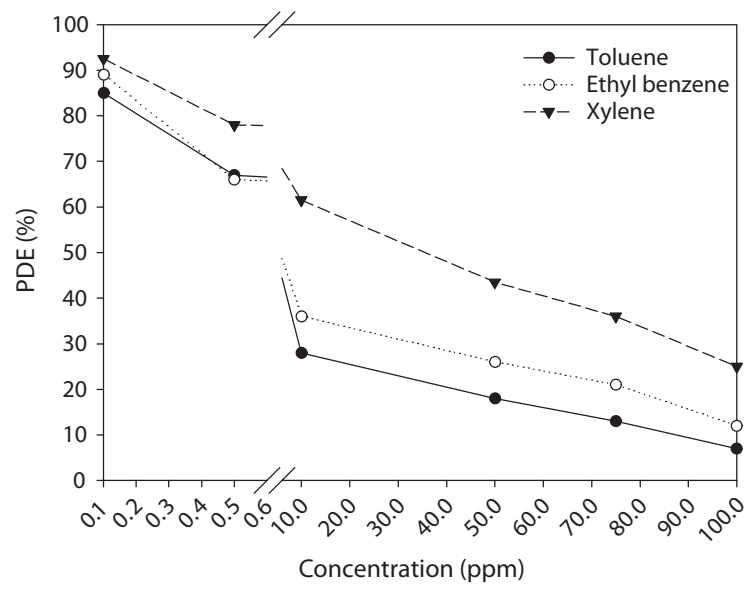

Fig. 8. Photocatalytic degradation efficiencies (PDEs, \%) of toluene, ethyl benzene and xylenes by the number of plates against initial concentrations. Efficiencies relative to a single cylinder-type reactor with six plates inserted whose surfaces were coated with $\mathrm{TiO}_{2}$.

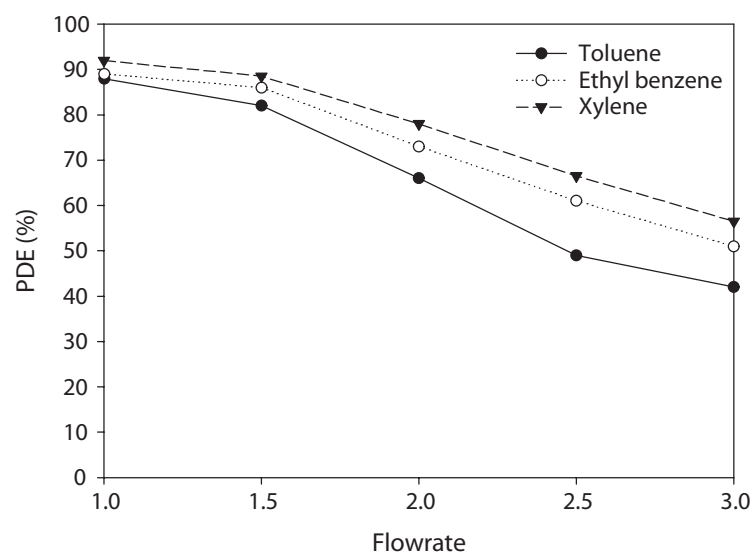

Fig. 9. Photocatalytic degradation efficiencies (PDEs, \%) of toluene, ethyl benzene and xylenes by the number of plates determined via a single cylinder-type reactor with inserted plates whose surfaces were coated with $\mathrm{TiO}_{2}$ against flow rates. likely due to a competitive adsorption rate between the contaminant molecules on the catalyst surface, since this adsorption rate is an important parameter with respect to the photocatalytic degradation efficiency $[25,26]$. The adsorption competition would be even greater at higher ICs. This suggests that, although the surface area of the catalyst was increased for the surfaceenhanced photocatalytic reactors, the adsorption rate is still an important parameter for the photocatalytic removal mechanism of the inserted plate photocatalytic reactors.

Fig. 9 illustrates the effects of FRs on degradation efficiencies of the target hydrocarbons. FR is closely associated with the residence time and face velocity in the reactors [27-29]. The relationships between degradation efficiencies and FRs for toluene, ethyl benzene and xylenes respectively, are as follows:

$\begin{array}{ll}\text { PDE (toluene })=69.2 / \mathrm{FR}+25.3 & \left(\mathrm{R}^{2}=0.85, \mathrm{p}<0.05\right) \\ \mathrm{PDE}(\text { ethyl benzene })=54.4 / \mathrm{FR}+40.4 & \left(\mathrm{R}^{2}=0.80, \mathrm{p}<0.05\right) \\ \mathrm{PDE}(\text { xylenes })=50.0 / \mathrm{FR}+47.3 & \left(\mathrm{R}^{2}=0.80, \mathrm{p}<0.05\right)\end{array}$

The degradation efficiency of all of the compounds decreased as the FR increased. As the FR increased from 1.0 to 3.0 $\mathrm{L} \mathrm{min}^{-1}$, the average degradation efficiencies obtained from the 6-hr PCO process in the 6 plate inserted photocatalytic reactor decreased from $87 \%$ to $42 \%$, from $89 \%$ to $52 \%$ and from $91 \%$ to $57 \%$ for toluene, ethyl benzene and xylenes respectively. The lower degradation efficiencies for the target compounds at the high flow rates are most likely due to an insufficient reactor residence time for these pollutants to transfer from the gas phase to the catalyst surface $[28,29]$. This assertion is supported by finding that the residence times in the present study, which were calculated by dividing the reactor volume by the flow rate, were $39.7,26.5,19.9,15.9,15.9$ and $13.2 \mathrm{sec}$ for flow rates of 1.0, 1.5, 2.0, 2.5 and $3.0 \mathrm{~L} \mathrm{~min}^{-1}$ respectively. As such, it is suggested that similar to no-plate inserted photocatalytic reactors [28, 29], the FR is still an important parameter for the photocatalytic removal mechanism in plate-inserted photocatalytic units.

\section{Conclusions}

The present study evaluated enhanced surface areas for cylindrical continuous-flow photocatalytic reactors on the degradation of three selected aromatic hydrocarbons (toluene, ethyl benzene, and xylenes). This purpose was achieved by comparing two sets of two reactors, a single cylindrical-type reactor without plates with a double cylinder-type reactor and a single cylindrical-type reactor without plates with a single cylindricaltype reactor with six inserted plates. These were compared for their photocatalytic activities on the target compounds. With regards to these pollutants, the degradation efficiencies were significantly or slightly higher for the single cylinder-type reactor than for the double cylinder-type reactor which had a greater catalytic surface area. This result suggests that the surface area which was enhanced for the double cylinder-type reactor would not elevate the photocatalytic degradation efficiency of the target compounds. However, it was found that for all of the target compounds, the degradation efficiencies increased gradually when the number of plates was increased, thereby suggesting that the surface area, when enhanced in the plate-inserted reactor would effectively elevate the photocatalytic degradation efficiency. In addition, this study confirmed that under the experimental conditions used in the present study, both IC and FR 
were important parameters in the photocatalytic removal mechanism of these plate-inserted photocatalytic reactors. However, it is noteworthy that different IC or FR dependency for the aromatic hydrocarbon degradation efficiency can be found under other experimental conditions.

\section{References}

1. Liu J, Mu Y, Zhang Y, et al. Atmospheric levels of BTEX compounds during the 2008 Olympic Games in the urban area of Beijing. Sci. Total Environ. 2009;408:109-116.

2. Choi SW. Characteristic of BTEX concentration ratio of VOC emission sources and ambient air in Daegu. J. Environ. Sci. 2007;16:415-423.

3. Office of Environmental Health Hazard Assessment. Proposition 65 status report safe habor levels: no significant risk levels for carcinogens and maximum allowable dose levels for chemicals causing reproductive toxicity--August 2005. San Francisco: California Environmental Protection Agency; 2005.

4. U.S. Environmental Protection Agency (EPA). National emissions standards for hazardous air pollutants: municipal solid waste landfills. Report 68/FR/2227. Washington, DC: EPA, Office of Air Quality Planning and Standards; 2003.

5. Revilla AS, Pestana CR, Pardo-Andreu GL, et al. Potential toxicity of toluene and xylene evoked by mitochondrial uncoupling. Toxicol. In Vitro 2007;21:782-788.

6. Chen CS, Hseu YC, Liang SH, Kuo JY, Chen SC. Assessment of genotoxicity of methyl-tert-butyl ether, benzene, toluene, ethylbenzene, and xylene to human lymphocytes using comet assay. J. Hazard. Mater. 2008;153:351-356.

7. Zou L, Luo Y, Hooper M, Hu E. Removal of VOCs by photocatalysis process using adsorption enhanced $\mathrm{TiO}_{2}-\mathrm{SiO}_{2}$ catalyst. Chem. Eng. Process. 2006;45:959-964

8. Son HJ, Jung CW, Bae SD. Photocatalytic degradation of algae and its by-product using rotating photocatalytic oxidation disk reactor. Environ. Eng. Res. 2009;14:170-173.

9. Zhang FJ, Chen ML, Oh WC. Characterization of $\mathrm{CNT} / \mathrm{TiO}_{2}$ elecrode prepared through impregnation with TNB and their phtoelectrocatalytic properties. Environ. Eng. Res. 2009; 14:32-40.

10. Gaya UI, Abdullah AH. Heterogeneous photocatalytic degradation of organic contaminants over titanium dioxide: a review of fundamentals, progress and problems. J. Photochem. Photobiol. C: Photochem. Rev. 2008;9:1-12.

11. Van Gerven T, Mul G, Moulijn J, Stankiewicz A. A review of intensification of photocatalytic processes. Chem. Eng. Process. 2007;46:781-789.

12. Mo J, Zhang Y, Yang R, Xu Q. Influence of fins on formaldehyde removal in annular photocatalytic reactors. Build. Environ. 2008;43:238-245.

13. Biard PF, Bouzaza A, Wolbert D. Photocatalytic degradation of two volatile fatty acids in monocomponent and multicomponent systems: comparison between batch and annular photoreactors. Appl. Catal. B: Environ. 2007;74:187-196.
14. Hossain MM, Raupp GB, Hay SO, Obee TN. Three-dimensional developing flow model for photocatalytic monolith reactors. AlChE J. 1999;45:1309-1321.

15. Nam W, Kim J, Han G. Photocatalytic oxidation of methyl orange in a three-phase fluidized bed reactor. Chemosphere 2002;47:1019-1024.

16. Na K, Kim YP, Moon I, Moon KC. Chemical composition of major VOC emission sources in the Seoul atmosphere. Chemosphere 2004;55:585-594.

17. Hung WC, Fu SH, Tseng JJ, Chu H, Ko TH. Study on photocatalytic degradation of gaseous dichloromethane using pure and iron ion-doped $\mathrm{TiO}_{2}$ prepared by the sol-gel method. Chemosphere 2007;66:2142-2151.

18. Liu B, Wen L, Zhao X. The surface change of $\mathrm{TiO}_{2}$ film induced by UV illumination and the effects on UV-vis transmission spectra. Appl. Surf. Sci. 2008;255:2752-2758.

19. Dvoranová D, Brezová V, Mazúr M, Malati MA. Investigations of metal-doped titanium dioxide photocatalysts. Appl. Catal. B: Environ. 2002;37:91-105.

20. Mejía MI, Marín JM, Restrepo G, Rios LA, Pulgarín C, Kiwi J. Preparation, testing and performance of a $\mathrm{TiO}_{2} /$ polyester photocatalyst for the degradation of gaseous methanol. Appl. Catal. B: Environ. 2010;94:166-172.

21. Jensen H, Soloviev A, Li Z, Sogaard EG. XPS and FTIR investigation of the surface properties of different prepared titania nano-powders. Appl. Surf. Sci. 2005;246:239-249.

22. Viana MM, Soares VF, Mohallem NDS. Synthesis and characterization of $\mathrm{TiO}_{2}$ nanoparticles. Ceram. Int. 2010;36:20472053.

23. Strini A, Cassese S, Schiavi L. Measurement of benzene, toluene, ethylbenzene and o-xylene gas phase photodegradation by titanium dioxide dispersed in cementitious materials using a mixed flow reactor. Appl. Catal. B: Environ. 2005;61:90-97.

24. Vildozo D, Ferronato C, Sleiman M, Chovelon JM. Photocatalytic treatment of indoor air: optimization of 2-propanol removal using a response surface methodology (RSM). Appl. Catal. B: Environ. 2010;94:303-310.

25. Xu W, Raftery D, Francisco JS. Effect of irradiation sources and oxygen concentration on the photocatalytic oxidation of 2-propanol and acetone studied by in situ FTIR. J. Phys. Chem. B 2003;107:4537-4544.

26. Chang CP, Chen JN, Lu MC. Characteristics of photocatalytic oxidation of gaseous 2-propanol using thin-film $\mathrm{TiO}_{2}$ photocatalyst. J. Chem. Technol. Biotechnol. 2004;79:1293-1300.

27. Zhao J, Yang X. Photocatalytic oxidation for indoor air purification: a literature review. Build. Environ. 2003;38:645-654.

28. Boulinguiez B, Bouzaza A, Merabet S, Wolbert D. Photocatalytic degradation of ammonia and butyric acid in plugflow reactor: degradation kinetic modeling with contribution of mass transfer. J. Photochem. Photobiol. A: Chem. 2008;200:254-261

29. Biard PF, Bouzaza A, Wolbert D. Photocatalytic degradation of two volatile fatty acids in an annular plug-flow reactor; kinetic modeling and contribution of mass transfer rate. Environ. Sci. Technol. 2007;41:2908-2914. 